\title{
Lingual clamping procedures for measuring oral vibrotactile thresholds: II. Effects of using a lower clamping disk
}

\author{
DONALD J. FUCCI and MICHAEL A. CRARY \\ School of Hearing and Speech Sciences, Ohio University, Athens, Ohio 45701 \\ and \\ KAL M. TELAGE \\ Department of Speech Pathology and Audiology, Ithaca College, Ithaca, New York 14850
}

\begin{abstract}
Lingual vibrotactile thresholds were obtained from four experimental groups using different clamping procedures. The purpose of the study was to evaluate the effects on sensitivity which occur when using a solid lower clamping disk vs. a ring-type bottom support. Findings indicated more sensitive thresholds across five frequencies for the conditions employing a solid lower clamping disk. Clamping conditions which do not compress lingual tissue also demonstrated differential sensitivity across frequencies. Implications of these results are discussed in reference to prior research concerned with the nature of lingual mechanoreceptors.
\end{abstract}

Research into the nature of lingual vibratory sensitivity has produced conflicting data. Sherrick (1953) reported that the dorsal lingual surface was selectively responsive to test frequencies from 40 to $1,200 \mathrm{~Hz}$, with maximum sensitivity being demonstrated at approximately $200-250 \mathrm{~Hz}$. These results conformed to previous data from nonoral structures (Sherrick, 1953). Verrillo (1966) questioned the findings of Sherrick on the basis that the dorsal lingual surface is void of the type of sensory receptors necessary for a frequency-selective response to vibratory stimuli. To support his contention, Verrillo compared vibratory sensory data obtained from the palmar surface to data obtained from the dorsal lingual surface. His results directly conflicted with those obtained by Sherrick (1953). Verrillo (1966) reported a flat frequency curve for the lingual surface and a U-shaped curve for the palmar surface. The discrepancy between these data has been explained by Verrillo in terms of Sherrick's failure to utilize a free surround disk to limit stimulus spread from the dorsal lingual surface to the ventral lingual surface which possesses frequency-sensitive receptors.

More recent investigations have produced results in agreement with Sherrick's (1953) observation of a frequency-selective response on the dorsal lingual surface (Telage \& Fucci, 1973, 1974; Telage, Fucci, $\&$ Arnst, 1972). All of these recent investigations utilized a free surround disk in obtaining lingual vibrotactile thresholds. They also used a solid lower clamping disk as part of a standard testing procedure (Figure 1). It is the purpose of the present investigation to study further the effects on sensitivity which occur when using a solid lower clamping disk and the potential role of the solid lower clamping disk in obtaining a frequency-selective response from the dorsal lingual surface.

\section{METHOD}

\section{Subjects}

Forty subjects, divided into four groups of 10 subjects in each group, comprised the experimental population. The mean age was 21 years and all subjects reported no history of hearing, speech, or sensorimotor disorders. Lingual vibrotactile thresholds were obtained for each group using one of four experimental conditions.

\section{Apparatus}

The equipment used in this study has been described in a previous publication (Fucci \& Kelly, 1972). The stimulus unit included a sine-wave generator, frequency counter, electronic switch-interval timer, amplifier, variable attenuator, and electromagnetic vibrator. The pulsed vibratory signal generated had a $50 \%$ duty cycle (on $1 / 2$ sec and off $1 / 2 \mathrm{sec}$ ) with a rise and decay time of $100 \mathrm{msec}$. The measurement unit consisted of an accelerometer, cathode follower, microphone amplifier, and voltmeter. A white-noise generator was used to present auditory masking at 80-85 dB HTL to subjects through TDH-39 headphones (Figure 1).

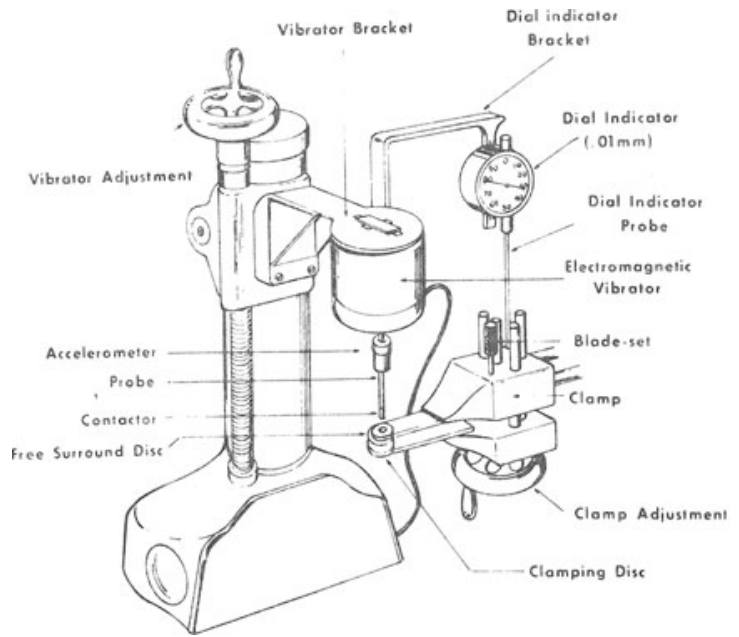

Figure 1. A schematic diagram of the vibrator and clamp assembly portion of the vibrotactile stimulator. 

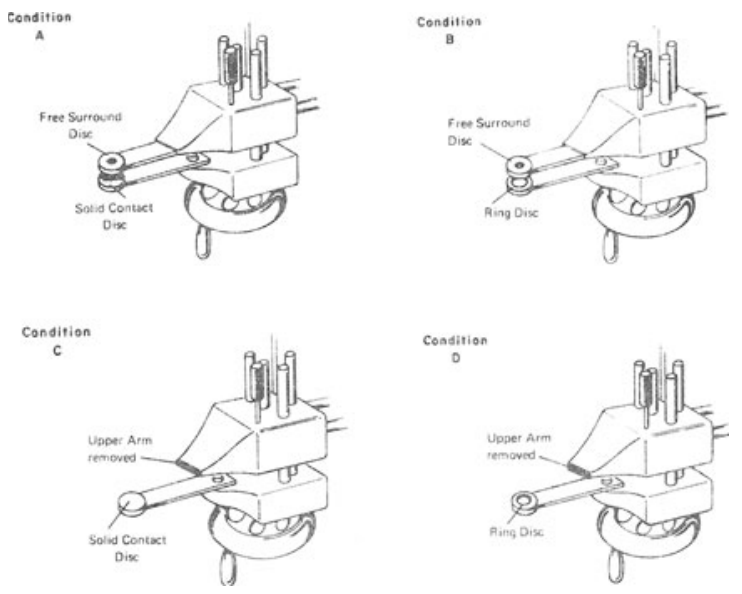

Figure 2. Schematic drawings showing the four clamping conditions used in the present investigation.

\section{Procedure}

Condition A represents the common clamping procedure used for lingual vibrotactile measures and includes an upper free surround disk and a solid lower clamping disk (Figure 2). Condition B provides for retention of the free surround disk, but substitution of a specialized rubber ring disk for the solid lower clamping disk (Figure 2). The ring disk is designed to minimize upward pressure on ventral lingual tissue. Since the lower clamping disk may be an independent variable affecting lingual responsiveness, the ring disk is used as a control procedure to better assess the influence of the free surround disk on threshold sensitivity. Condition $C$ provides for retention of the lower clamping disk but the elimination of the free surround disk, producing an experimental situation which does not restrict stimulus spread and limits interaction from the lower clamping disk since the tongue is not clamped (Figure 2). Condition D provides for the elimination of the free surround disk and substitution of the ring disk for the lower clamping disk (Figure 2). This condition is designed to provide for stimulus spread while maximally limiting upward compression of ventral mucocutaneous tissues.

Lingual vibrotactile thresholds were obtained for each group at frequencies of $100,200,250,300$, and $400 \mathrm{~Hz}$, using an ascending psychophysical method of limits. Frequencies were randomized to cancel out any possible extraneous variables. A median of three millivolt threshold trials was accepted as a subject's lingual threshold. These data were later converted to peak displacement in microns by using a standard $g$ formula for acceleration conversion.

Subjects were seated in a dental chair and extended their tongues either on or between the clamping disks as prescribed by the experimental condition used. During Conditions $A$ and $B$, the tongue was clamped tightly enough to assist in maintaining its position while still permitting easy withdrawal. The stimulus was applied either directly or through the free surround disk. The contactor area was $.128 \mathrm{~cm}^{2}$ in all conditions and, when the free surround disk was used, the area around the contactor was $1 \mathrm{~mm}$ (Figure 1).

\section{RESULTS AND DISCUSSION}

Threshold results for test conditions are presented in Table 1. Data reflecting the influence of the lower clamping disk are summarized for Conditions A-B, in which the free surround disk was held constant, and for Conditions C-D, where no free surround disk was used. The data are compared in a manner which attempts to isolate the possible influence of the free surround disk on threshold sensitivity. In this manner, any effect of the lower clamping disk vs. the lower ring disk is observable.

An assessment of the data indicates that, in each condition where a lower clamping disk was used, as opposed to a lower ring disk, greater sensitivity was observed. As anticipated, the lower clamping disk used in conjunction with the free surround disk produced the most sensitive threshold data. The least sensitive thresholds occurred when the ring disk was used without a free surround disk. A selective frequency response was maintained regardless of the clamping condition.

Utilization of a solid lower clamping disk does not appear to be a factor in obtaining a frequency-selective response from the dorsal lingual surface. This procedure does, however, have a noticeable effect on the sensitivity of reported thresholds. In fact, lower thresholds are demonstrated in both comparative conditions where a complete clamping assembly was used (Conditions $\mathrm{A}$ and B). One explanation may be that using both an upper and a lower clamping disk provides needed support for the lingual mass to maintain a stable position in reference to the contactor during stimulation. Lack of such support may result in uncontrolled tongue movements which could reduce the reported threshold values, as evidenced in Conditions $\mathrm{C}$ and D. It appears that the more efficient clamping device, in terms of sensitivity, is that which supplies the most support to the lingual structure. This is represented by Condition $\mathbf{A}$ in the present study.

From these data two observations can be made. First, the lingual dorsal surface demonstrates a frequency-specific response to vibratory stimuli regardless of the clamping procedure used. Second, while the

Table 1

A Comparison of Mean Threshold Differences in Microns of Peak Displacement Between the Lower Clamping Disk and Ring Disk Conditions

\begin{tabular}{|c|c|c|c|c|c|c|}
\hline & \multirow[b]{2}{*}{ Conditions } & \multicolumn{5}{|c|}{ Frequencies $(\mathrm{Hz})$} \\
\hline & & 100 & 200 & 250 & 300 & 400 \\
\hline $\begin{array}{l}\text { B } \\
\mathbf{A}\end{array}$ & $\begin{array}{l}\text { Ring Disk/Free Surround Disk } \\
\text { Lower Clamping Disk/Free Surround Disk } \\
\text { Difference }\end{array}$ & $\begin{array}{r}5.3 \\
4.8 \\
.5\end{array}$ & $\begin{array}{r}2.8 \\
2.6 \\
.2\end{array}$ & $\begin{array}{r}2.1 \\
1.7 \\
.4\end{array}$ & $\begin{array}{r}1.5 \\
1.3 \\
.2\end{array}$ & $\begin{array}{l}.9 \\
.7 \\
.2\end{array}$ \\
\hline $\begin{array}{l}\mathrm{D} \\
\mathrm{C}\end{array}$ & $\begin{array}{l}\text { Ring Disk/No Free Surround } \\
\text { Lower Clamping Disk/No Free Surround } \\
\text { Difference }\end{array}$ & $\begin{array}{l}7.7 \\
6.7 \\
1.0\end{array}$ & $\begin{array}{r}3.2 \\
2.6 \\
.6\end{array}$ & $\begin{array}{r}2.2 \\
1.8 \\
.4\end{array}$ & $\begin{array}{r}2.0 \\
1.7 \\
.3\end{array}$ & $\begin{array}{r}1.7 \\
1.1 \\
.6\end{array}$ \\
\hline
\end{tabular}


solid lower clamping disk demonstrates no effect on the frequency-specificity of the response, it does have a noted effect on the degree of sensitivity at each frequency. For all test frequencies, the combination of free surround disk and solid lower clamping disk produces the most sensitive thresholds.

\section{REFERENCES}

FuCCI, D., \& Kelly, D. H. New instrumentation for research on vibrotactile sensitivity of the tongue. Review of Scientific Instrumentation, 1972, 43, 1748-1751.

SHERRICK, C. W. Variables affecting sensitivity of the skin to mechanical vibration. Journal of Experimental Psychology, 1953, 45, 273-282.
TelaGe, K. M., \& Fucci, D. Vibrotactile stimulation: A future clinical tool for speech pathologists. Journal of Speech and Hearing Disorders, 1973, 38, 442-447.

Telage, K. M., \& FuccI, D. Concerning intrasubject measurements of successive lingual vibrotactile responses. Perceptual and Motor Skills, 1974, 39, 1047-1052.

Telage, K. M., Fucci, D., \& ARnst, D. J. Normative study of oral vibrotactile sensitivity. Perceptual and Motor Skills, 1972, 35, 671-676.

Verrillo, R. T. Specificity of a cutaneous receptor. Perception \& Psychophysics, 1966, 1, 149-153.

(Received for publication August 12, 1977.) 